\title{
Cardiac abnormalities detected by echocardiography and cardiac magnetic resonance in healthcare professionals recovered from non-severe COVID-19
}

\author{
Jadwiga Fijałkowska', Jakub Sobolewski², Anna Glińska', Izabela Pisowodzka², Radosław Nowak², \\ Marta Żarczynska-Buchowiecka³, Joanna Pieńkowska', Marcin Gruchała², Edyta Szurowska', \\ Karolina Dorniak ${ }^{3}$, Marcin Fijałkowski \\ 'Department of Radiology, Medical University of Gdansk, Gdańsk, Poland \\ ${ }^{2} 1^{\text {st }}$ Department of Cardiology, Medical University of Gdansk, Gdańsk, Poland \\ ${ }^{3}$ Department of Noninvasive Cardiac Diagnostics, Medical University of Gdansk, Gdańsk, Poland
}

Correspondence to: Marcin Fijałkowski, MD, PhD, $1^{\text {st }}$ Department of Cardiology Medical University of Gdansk Dębinki 7, 80-211 Gdańsk,

Poland,

phone: +485858447 10,

e-mail:

marcin.fijalkowski@gumed.edu.pl

Copyright by the Author(s), 2021

Kardiol Pol. 2021;

79 (11): 1256-1258;

DOI: $10.33963 /$ KP.a2021.0114

Received:

July 12, 2021

Revision accepted:

September 20, 2021

Published online:

September 20, 2021

\section{INTRODUCTION}

Patients with COVID-19 typically present with symptoms and signs of respiratory tract infection. Cardiac manifestations have also been described, however, the prevalence and characteristics of myocardial injury due to COVID-19 are still unknown [1].

Healthcare professionals are at the highest personal risk of infection. The majority of healthcare professionals have undergone infection with no or mild to moderate symptoms. However, some studies suggest the presence of myocardial inflammatory process after recovery from COVID-19 infection even in asymptomatic or mildly symptomatic patients [2]. The impact of COVID-19 disease on this particularly large group of patients should be fully elucidated, as the long-term effect on the health status of healthcare professionals remains important for the maintenance of the healthcare system.

Our objective was to characterize myocardial injury in healthcare professionals recovered from non-severe COVID-19 as detected by transthoracic echocardiography (TTE) and cardiac magnetic resonance (CMR).

\section{METHODS}

We enrolled 95 healthcare workers who survived COVID-19 confirmed in a reverse transcription-polymerase chain reaction (PCR) swab test. They included medical doctors, nurses, and paramedics who have been working at the hospital during the pandemic. We performed TTE and CMR up to 4 months from the diagnosis of the SARS-CoV-2 infection. TTE and CMR were performed from December 2020 to April 2021. No significant ST/T changes on the standard 12-lead electrocardiogram were noted. The Local Ethics Committee approved the study protocol, and written informed consent was obtained from all the participants.

Transthoracic echocardiography was performed according to the Working Group on Echocardiography of the Polish Cardiac Society using VIVID E95 (GE Ultrasound, Horten, Norway) [3]. Traditional echocardiographic measurements were obtained, and additionally, global right (RV) and left ventricle (LV) longitudinal peak systolic strain (GLPS) was quantified.

CMRwas performed on a 1.5Tscanner(Magnetom Sola, Siemens, Munich, Deutschland) using a comprehensive standardized protocol for myocarditis including steady state free precession cine for function evaluation, $\mathrm{T} 1$ and T2 mapping, extracellular volume fraction (ECV), and late gadolinium enhancement (LGE). All parameters were analyzed for $12 \mathrm{LV}$ segments ( 6 basal and 6 mid-ventricular) - a total of 1068 segments were evaluated and compared to the institutional scanner-specific reference range. Three experienced cardiologists and two radiologists reviewed echocardiography and CMR examinations. 


\section{Statistical analysis}

Continuous data are presented as means (standard deviation [SD]) for normally distributed variables while categorical data are expressed in proportion. The Shapiro-Wilk test was performed to assess the normality of the distribution of continuous data. Computations were performed using Statistica version 13.3. A $P$-value $<0.05$ was considered to be statistically significant.

\section{RESULTS AND DISCUSSION}

The mean time between COVID-19 diagnosis and CMR was 72 (30) days (range, 22-126 days). Six subjects were excluded from the study due to technical issues (poor acoustic window -1 , inadequate breath-holding -2 , or severe artifacts - 3). Eighty-nine were included in the study: 69 (77\%) females, mean age 42 (10) years, mean BMI 26 (4) $\mathrm{kg} / \mathrm{m}^{2}$, with no previous significant cardio-vascular disorders, 17 (19\%) patients had well-controlled arterial hypertension, 2 had hyperlipidemia and one had diabetes mellitus. None of the enrolled subjects required hospitalization for the SARS-CoV-2 infection, 57 (64\%) patients suffered from moderate symptoms and $32(36 \%)$ had mild or no symptoms.

TTE: mean left ventricular ejection fraction (LVEF) was 57 (5)\%; range 38\%-70\%, mean GLPS was -19.6 (2.3)\%; range $12.7 \%-22.7 \%$. Two patients (2.2\%) had an enlarged LV. Right ventricle (RV) parameters: RVID 33 (3.9) mm, TAPSE 22.6 (2.2) $\mathrm{mm}, \mathrm{S}^{\prime} 11.6(1.3) \mathrm{cm} / \mathrm{s}$ and RV free wall 27.9 (4.0)\%.

CMR: mean LVEF was 60 (5)\%; range $45 \%-70 \%$ but $29 \%$ subjects had LVEF below lower limit for age and sex. Mean EDV was 144 (27) ml, and mean ESV was 60 (15) ml. Six patients $(6.7 \%)$ had an enlarged LV.

Native T1 mapping: 29 (32\%) patients had at least one segment with native T1 elevation (mean 6 [2] segments per patient), mostly in the septum and infero-lateral wall. In total, native T1 was elevated in 185 of 1068 segments (17\%). Eight patients (9\%) had elevated mean T1 calculated for all $12 \mathrm{LV}$ segments. Mean native T1 for the entire cohort was 1003 (23) ms (range: 959-1056 ms) while the scanner-specific reference range in our institution in healthy subjects is $951-1035 \mathrm{~ms}$.

T2 mapping: 35 (39\%) patients had at least one segment with T2 elevation (mean 6.5 [2.5] segments per patient), mostly in the septum and basal and mid-ventricular segment of the infero-lateral wall. A total of 228 segments (21\%) had elevated T2. Seventeen patients (19\%) had elevated mean T2 calculated for all $12 \mathrm{LV}$ segments. Mean T2 for the entire cohort was 45 (3) ms (range: 38-54 ms), scanner-specific reference range in our institution in healthy subjects was 39-49 ms.

ECV analysis: 30 (34\%) patients had at least one segment with high ECV, (mean 9 [3] segments per patient), mostly in the septum and basal and mid-ventricular segment of the infero-lateral wall. A total of 269 segments (25\%) had elevated ECV. Eight patients (9\%) had high mean ECV calculated for all 12 LV segments, mean ECV was 26.5 (2.7)\% (range: $20 \%-39.8 \%$ ) while the reference range in our institution in healthy subjects was $22 \%-30 \%$.

LGE analysis: 51 subjects (57\%) had at least one segment with LGE, mean 1.9 (1.1) LGE segments (range: 1-6 segments) were involved per patient, with a predominance of the basal and midventricular infero-lateral segments. Positive LGE was found in a total of 93 segments (9\%), mostly epicardial or midwall (i.e. non-ischemic) patterns.

Three patients (3.5\%) fulfilled the original Lake Louise Criteria for myocardial injury due to active inflammatory process: co-existing myocardial edema evaluated by STIR and LGE (+) for the same region. Two of these patients had symptoms (mild dyspnea on exertion).

The updated Lake Louise criteria based on parametric mapping (i.e. elevated $\mathrm{T} 2$ and at least one of the T1-based criteria including elevated native $\mathrm{T} 1$, high ECV, or LGE) were positive in 14 (15\%) patients (Figure 1). The majority of these patients were asymptomatic.

Myocardial injury detected by TTE and CMR among healthcare professionals, convalescents from non-severe COVID-19 has not been fully evaluated so far [4, 5]. Left ventricular ejection fraction was decreased in $29 \%$ of cases, assessed by both TTE and CMR, and LV longitudinal myocardial function was also slightly decreased in $39 \%$ of the subjects. CMR identified $32 \%$ of patients with elevated native T1 (a nonspecific finding seen with acute myocardial injury/edema, fibrosis, or infiltration), $39 \%$ with elevated T2 (a marker of acute myocardial injury/edema), and 57\% with LGE (a marker of acute myocardial injury, fibrosis, or scar). To date, only a few CMR studies that evaluated COVID-19-related myocardial injury in several patient groups were published and the findings are ambiguous. For example, in competitive student athletes, the prevalence of myocarditis was very low (1.4\%). On the other hand, in an unselected cohort of 100 patients, tissue characterization abnormalities were common (raised native $\mathrm{T} 1$ in $73 \%$ cases, raised $\mathrm{T} 2$ in $60 \%$ cases, LGE $32 \%)[1,2]$. In another study evaluating 26 athletes, in line with our results, $15 \%$ of participants had findings consistent with myocarditis and $46 \%$ had LGE [6]. Our results are also in agreement with findings of another study on 139 healthcare workers with a confirmed past SARS-CoV-2 infection, where cardiac magnetic resonance features of myocarditis were observed in $37 \%$ of the participants at a median of 10 weeks after infection [4]. According to the latest meta-analysis, nearly half of recovered COVID-19 patients exhibited one or more abnormal CMR findings, but it is still debatable if CMR should be a frontline and widespread screening tool, or rahter than a clinically indicated and selective downstream test as part of cardiovascular risk stratification [7, 8].

Our study has some limitations. There is no control group to evaluate CMR changes among healthy subjects. Baseline CMR to compare or a serum laboratory test, such as troponin level, were not obtained. Furthermore, detection of mild myocardial injury may have been missed because the optimal 


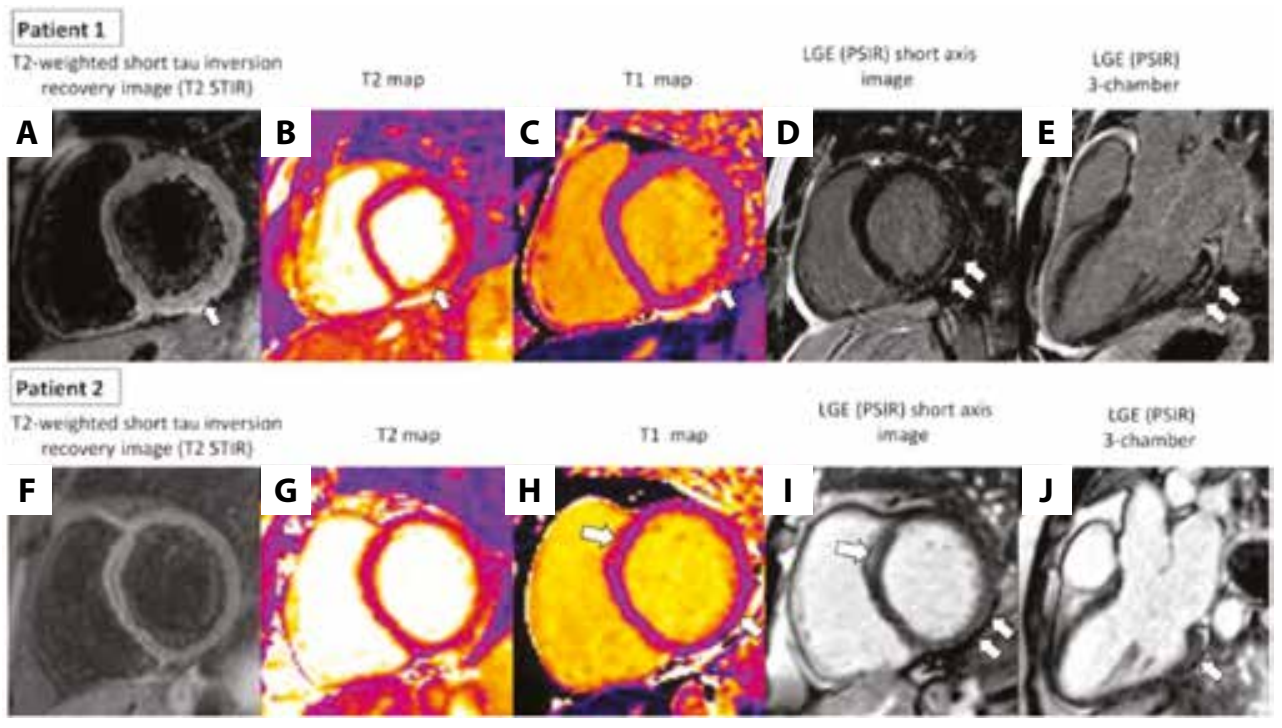

Figure 1. Patient 1. Cardiac magnetic resonance (CMR) findings consistent with acute inflammatory injury. A. Basal short axis T2-weighted short tau inversion recovery image (T2 STIR). B. Basal short axis T2 map with locally elevated T2 values. Both $\mathbf{A}$ and $\mathbf{B}$ indicated edema in the basal infero-lateral segment (arrows). C. Basal short axis native T1 map with locally elevated T1 values (arrows). D. Basal short axis. E. 3-chamber long axis late gadolinium enhancement images, indicating irreversible myocardial injury/fibrosis of the infero-lateral segment (phase-sensitive inversion recovery sequence; arrows). Patient 2. CMR findings consistent with non-acute myocardial injury: T2-weighted short tau inversion recovery image (T2 STIR; F) and T2 map (G) showed no abnormalities (arrows). H. Basal short axis native T1 map showed slightly increased local native T1 values limited to areas where irreversible myocardial injury/fibrosis for basal infero-lateral (arrows) and basal anteroseptal segments (arrows) a was demonstrated on late gadolinium enhancement images (phase-sensitive inversion recovery sequence), in the basal short axis (I) and in the 3-chamber long axis (J)

aThe apparent late gadolinium enhancement area in the anteroseptal segment can be an artifact related either to the proximity of the aortic root or to the contrast media lingering in the RV septal trabeculations and should be interpreted with caution

time to perform CMR appears to be unknown. It should be underlined that the interpretation of LGE images is, to some extent, subjective. However, our standard protocol includes re-acquisition of the suspected LGE lesions in a plane perpendicular to the index plane, as well as re-acquisition using an alternative phase encoding direction.

To conclude, cardiac involvement revealed by CMR among healthcare professionals who recovered from a non-severe SARS-CoV-2 infection is common. These subjects need to be monitored, followed up and should not engage in physical activity. Further research is needed to demonstrate whether CMR evidence of myocardial injury after recovery from COVID 19 is associated with short- and long-term adverse outcomes in this particular cohort.

\section{Article information}

Funding: Medical University of Gdansk's Grant Program "Young Creator of Science" — part of the "Excellence Initiative - Research University" Program.

\section{Conflict of interests: None declared.}

Open access: This article is available in open access under Creative Common Attribution-Non-Commercial-No Derivatives 4.0 International (CC BY-NC-ND 4.0) license, allowing to download articles and share them with others as long as they credit the authors and the publisher, but without permission to change them in any way or use them commercially. For commercial use, please contact the journal office at kardiologiapolska@ptkardio.pl.

How to cite: Fijałkowska J, Sobolewski J, Glińska A, et al. Cardiac abnormalities detected by echocardiography and cardiac magnetic resonance in healthcare professionals recovered from non-severe COVID-19. Kardiol Pol. 2021; 79(11): 1256-1258, doi: 10.33963/KP.a2021.0114.

\section{REFERENCES}

1. Puntmann VO, Carerj ML, Wieters I, et al. Outcomes of cardiovascular magnetic resonance imaging in patients recently recovered from coronavirus disease 2019 (COVID-19). JAMA Cardiol. 2020; 5(11): 1265-1273, doi: 10.1001/jamacardio.2020.3557, indexed in Pubmed: 32730619.

2. Starekova J, Bluemke DA, Bradham WS, et al. Evaluation for myocarditis in competitive student athletes recovering from coronavirus disease 2019 with cardiac magnetic resonance imaging. JAMA Cardiol. 2021; 6(8): 945-950, doi: 10.1001/jamacardio.2020.7444, indexed in Pubmed: 33443537.

3. Gackowski A, Lipczyńska M, Lipiec P, et al. Review Committee, Reviewers (on behalf of the Polish Cardiac Society). Echocardiography during the coronavirus disease 2019 (COVID-19) pandemic: expert opinion of the Working Group on Echocardiography of the Polish Cardiac Society. Kardiol Pol. 2020; 78(4): 357-363, doi: 10.33963/KP.15265, indexed in Pubmed: 32241097.

4. Eiros R, Barreiro-Perez M, Martin-Garcia A, et al. Pericarditis and myocarditis long after SARS-CoV-2 infection: a cross-sectional descriptive study in health-care workers. MedRxiv. 2020, doi: 10.1101/2020.07.12.20151316.

5. Gasecka A, Pruc M, Kukula K, et al. Post-COVID-19 heart syndrome. Cardiol J. 2021; 28(2): 353-354, doi: 10.5603/CJ.a2021.0028, indexed in Pubmed: 33645626.

6. Rajpal S, Tong MS, Borchers J, et al. Cardiovascular magnetic resonance findings in competitive athletes recovering from COVID-19 infection. JAMA Cardiol. 2021; 6(1): 116-118, doi: 10.1001/jamacardio.2020.4916, indexed in Pubmed: 32915194

7. Terlecki M, Wojciechowska W, KlocekM, et al. Association between cardiovascular disease, cardiovascular drug therapy, and in-hospital outcomes in patients with COVID-19: data from a large single-center registry in Poland. Kardiol Pol. 2021; 79(7-8): 773-780, doi: 10.33963/KP.15990, indexed in Pubmed: 33926173

8. Kim JY, Han K, Suh YJ. Prevalence of abnormal cardiovascular magnetic resonance findings in recovered patients from COVID-19: a systematic review and meta-analysis.J Cardiovasc Magn Reson. 2021; 23(1): 100, doi: 10.1186/s12968-021-00792-7, indexed in Pubmed: 34479603. 